\title{
La politique étrangère des États fédéraux : cas belge et canadien
}

\author{
David Anciaux
}

Institut d'Études Européennes - Université Libre de Bruxelles

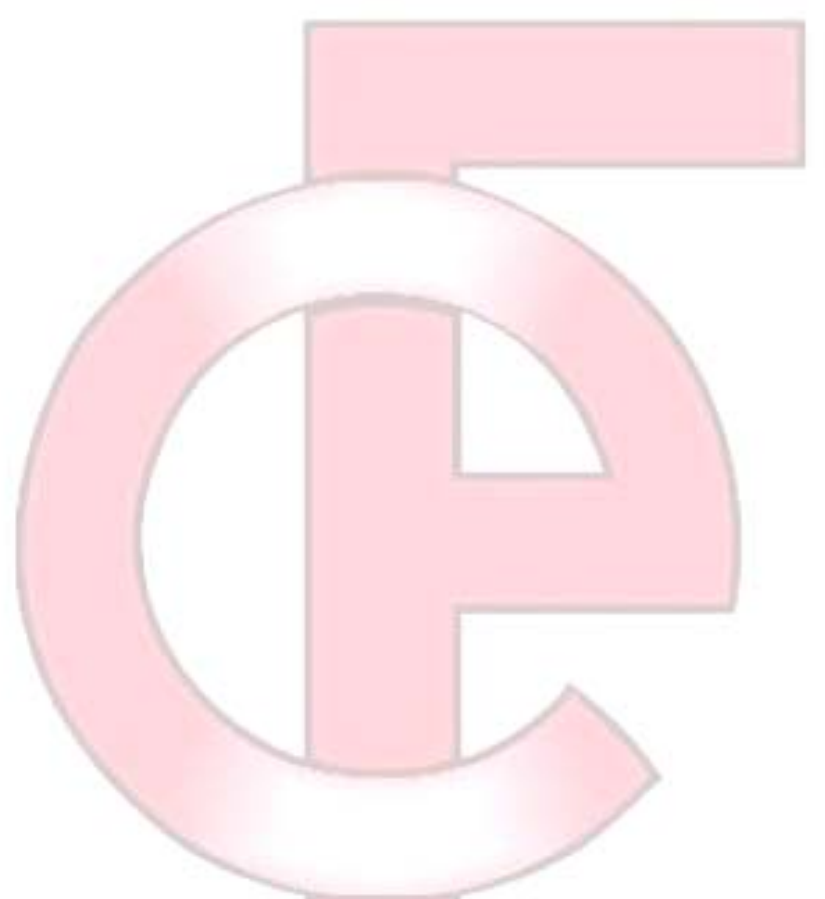

David Anciaux est un étudiant de « master » en études européennes - finalité « politique » - à l'Institut d'Études Européennes (IEE) de l'Université Libre de Bruxelles. Ses spécialisations portent notamment sur les domaines de la politique belge et européenne, les politiques étrangères belge et canadienne, le sentiment identitaire en Europe ainsi que la diplomatie culturelle. Ces spécialisations ont été permises grâce à des études en science politique effectuées, notamment lors d'un échange académique au Canada. David Anciaux est également éditeur francophone de la revue « Eyes on Europe » éditée à l'IEE. Ses remerciements vont à Monsieur Eric Remacle pour les conseils prodigués. 


\section{Introduction}

Depuis les années 70, plusieurs entités territoriales ont affirmé leur présence au niveau international. En entreprenant ces processus, elles ont remanié le concept traditionnel de souveraineté qui considérait la présence internationale comme émanant uniquement du pouvoir central. Cette présence des sous-entités territoriales à l'extérieur des frontières nationales est due à un nombre important de causes variant des raisons économiques aux raisons culturelles ou politiques ${ }^{1}$. Ces entités sont non seulement présentes sur la scène internationale mais également dans la formation de la politique étrangère des États fédéraux.

Le but de ce travail est d'analyser si différentes politiques étrangères sont possibles au sein des États fédéraux. Pour des raisons méthodologiques, deux approches vont être mises en œuvre dans le présent travail : la théorie de la gouvernance à plusieurs niveaux (multi-level governance approach) et l'approche centralisée (state-centric approach). Ces approches nous permettront de voir si plusieurs types de gouvernance sont possibles dans la politique étrangère de pays fédéraux. Notre recherche porte sur deux États : la Belgique et le Canada. Diverses raisons ont

\footnotetext{
${ }^{1}$ Pour plus dans ce domaine, lire DUCHACEK, I. D., “The International Dimension of Subnational Self-Government”, Publius, vol.14, N4, FALL 1984, pp.5-31
}

conduit à ce choix : Ces deux pays renferment des divisions linguistique et culturelle, ainsi qu'un mouvement séparatiste². À ce titre, il devient intéressant de voir comment ces deux pays gèrent les différences internes dans la politique étrangère. Les hypothèses du travail sont les suivantes : La Belgique correspond à un système de gouvernance à plusieurs niveaux tandis que le Canada correspond à une approche centralisée.

Pour cette analyse, le travail est divisé en trois chapitres. La première partie constitue un éclaircissement des deux approches utilisées selon les théories définies par Hooghe et Marks. Le chapitre suivant analyse la pertinence de la comparaison entre les deux États fédéraux étudiés. Cette partie explique brièvement le fonctionnement des deux États afin de faciliter la compréhension ultérieure. Le dernier chapitre se centre sur les similarités et les différences des deux façons d'établir la politique étrangère. Deux aspects de cette problématique y sont analysés : le pouvoir de conclure des traités et la représentation étrangère des entités fédérées.

\section{1- Approche théorique}

Deux approches de prises de décisions sont présentées : la gouvernance à plusieurs

\footnotetext{
${ }^{2}$ KARMIS, D., GAGNON, A-G., « Fédéralisme et identités collectives au Canada et en Belgique : des itinéraires différents, une fragmentation similaire », Canadian Journal of Political Science / Revue canadienne de science politique, Vol.29, N³, sept. 1996, pp. 435-468
} 
niveaux (multi-level governance approach) et l'approche centralisée (state-centric approach). Cette explication ne prétend pas être exhaustive mais vise à présenter les bases qui seront utiles à la compréhension et l'analyse générale de ce texte.

L'approche à plusieurs niveaux (multilevel governance ou MLG) a été créée par Marks et est utilisée principalement comme procédé analytique afin de saisir le système décisionnel de l'Union Européenne. Elle fut d'abord décrite comme un « système de négociation continue entre différents gouvernements situés à plusieurs niveaux territoriaux - supranational, national, régional et le local »3. La définition de cette approche pourrait être divisée en trois parties ${ }^{4}$ :

- Selon l'approche à plusieurs niveaux, les compétences de prises de décisions sont partagées par des acteurs dispersés à différents niveaux plutôt que concentrés au niveau fédéral ;

- Il y a une perte de contrôle, dans les gouvernements nationaux pris individuellement, en raison de la prise de décision collective entre les différents niveaux ;

- Les différents sièges de pouvoir (political arenas) sont interconnectés au

\footnotetext{
${ }^{3}$ HOOGHE, L., MARKS, G., "Unraveling the Central State, but How? Types of Multi-level Governance”, American Political science review, N97, 2003, p.3.

${ }^{4}$ HOOGHE, L., MARKS, G., Multi-Level Governance and European Integration, Lanham, Rowman \& Littlefield Publishers, 2001, p.3.
}

lieu d'être centralisés. Selon ce point de vue, les sous-entités nationales peuvent agir au niveau national et supranational. Donc, en plus d'être présents au niveau international, les États sont également des entités fédérées.

\section{Contrairement à l'approche de la} gouvernance à plusieurs niveaux, les éléments à la base du modèle centralisé affirment le rôle de l'État fédéral comme acteur majeur. Selon cette approche issue de la théorie « réaliste » des relations internationales, les acteurs fondamentaux sont les gouvernements nationaux qui agissent selon leurs principaux intérêts. Les partisans de cette théorie considèrent néanmoins l'influence des entités infranationales ou des acteurs économiques et sociaux dans la définition de la politique. Mais l'influence n'existe que dans le domaine interne, comme première étape à la conduite de la politique étrangère. Contrairement à l'approche à plusieurs niveaux, les adhérents de l'approche centralisée ne considèrent pas les différents sièges de pouvoir comme interconnectés, mais plutôt centralisés ${ }^{5}$.

\section{2- La comparabilité des cas belge et canadien}

Le Canada et la Belgique ont plusieurs caractéristiques communes. Ils sont tous les deux considérés comme pays regroupant deux langues, voire deux cultures différentes : Les

\footnotetext{
${ }^{5}$ HOOGHE, L., MARKS, G., Multi-Level Governance and European Integration, Loc. Cit., p.2
} 
francophones et les anglophones au Canada, en contraste aux francophones et néerlandophones en Belgique. En plus des deux langues principales de la Belgique, il existe une troisième région linguistique : les germanophones représentés dans le système fédéral par la Communauté germanophone. Cependant, cette communauté est relativement faible d'un point de vue numérique, ne représentant que 70000 personnes sur les 10 millions d'habitants que compte l'État belge. Par conséquent, nous aborderons peu le rôle de cette communauté sur la scène internationale.

La forme fédérale des deux pays est très différente tout comme leurs évolutions historiques. Alors que la fédération canadienne a été créée en 1867 avec l'adoption de l'Acte de l'Amérique du Nord britannique, la première étape vers le fédéralisme belge remonte seulement à 1970. Depuis, diverses réformes ont eu lieu en 1980, 1988, 1993 et 2001 auxquelles s'ajoutent des réformes de moindre ampleur qui ont transformé l'État unitaire d'avant 1970 en un système fédéral. Ces réformes ont créé un pays divisé en trois régions : la Région wallonne, la Région flamande et la Région Bruxelles-Capitale. Les régions sont compétentes sur ce qui touche au « sol » comme l'environnement, l'eau, l'électricité, l'économie, etc. La Belgique est également divisée en trois communautés : la
Communauté française ${ }^{6}$, la Communauté flamande et la Communauté germanophone. Les compétences des communautés sont dites « personnalisables ${ }^{7}$ avec des domaines comme la culture, l'éducation et partiellement le système de santé.

Avec moins de complexité que le système belge, le Canada est divisé en dix provinces et trois territoires. Les provinces sont très différentes les unes des autres à divers niveaux tels que politique, économique et social. L'Ontario et le Québec sont les deux provinces les plus peuplées avec respectivement huit et sept million d'habitants. L'Alberta, moins peuplée que les deux provinces précédentes, est la plus riche grâce à une économie forte basée sur l'industrie pétrolière, alors que la Colombie-Britannique a une économie plus axée sur les pays d'Asie Pacifique comparativement aux autres provinces. Selon la loi constitutionnelle de 1982, les provinces ont des compétences exclusives comme les hôpitaux, la propriété et le droit civil, les ressources et l'éducation.

Pour résumer les similitudes principales entre les deux systèmes fédéraux :

\footnotetext{
${ }^{6}$ Depuis 2000, la Communauté française a été renommé Communauté Wallonnie-Bruxelles. Mais, la référence à la Communauté française sera utilisée dans ce travail car ce nom est utilisé dans les institutions internationales et au sein de la fédération belge.

${ }^{7}$ Terme désignant que ces compétences touchent directement à la personne, domaine où la langue à une dimension prépondérante.
} 
- Les compétences des unités territoriales sont exclusives. L'État fédéral n'a donc pas le droit de s'immiscer dans les compétences spécifiquement attribuées aux entités fédérées ;

- Il n'existe pas de hiérarchie entre l'État fédéral et les entités fédérées ;

- Les compétences entre les différentes entités sont asymétriques. Au Canada, les compétences sont distribuées de manière similaire entre les provinces, mais toutes les entités ne décident pas de légiférer dans ces domaines. Par exemple, la politique d'immigration est la même pour tout le pays à l'exception particulière du Québec qui a décidé d'établir des procédures particulières pour l'attribution du visa. En Belgique, les différentes réformes ont introduit un système fortement asymétrique. Les institutions de la Communauté flamande exercent celles de la Région flamande. Des compétences attribuées à la Communauté française ont été données à la Région wallonne pour des raisons budgétaires ;

- Les deux pays sont divisés en entités fédérées selon une logique territoriale, plutôt qu'en fonction des simples caractéristiques personnelles. Les autorités constituées sont donc compétentes uniquement sur les territoires attribués et non sur les personnes ayant une spécificité similaire comme la langue ;

- Pour être applicables dans les États, les traités internationaux doivent faire l'objet d'une transposition en droit interne. Cette transposition doit être exécutée soit par les entités fédérées ou par l'État fédéral.

Les deux pays sont distincts sur plusieurs éléments :

- La Belgique est un fédéralisme de superposition. Les deux entités régions et communautés - se recouvrent. Par exemple, la ville de Tournai est située dans la Région Wallonne et dans la Communauté française ;

Les deux pays ont des systèmes politiques où les partis politiques sont importants. Au Canada, la majorité des partis se présentant aux élections fédérales couvre tout le pays (à l'exception du Bloc québécois et de quelques petits partis). En Belgique, les partis couvrent uniquement leurs régions linguistiques ;

- Au niveau international, les entités fédérées belges ont le pouvoir de négocier et de conclure des traités pour leurs compétences explicites tandis que ce pouvoir est strictement réservé à Ottawa pour le Canada. Ce point sera 
examiné plus précisément dans les chapitres suivants.

\section{3- Les États fédéraux à l'étranger}

Perçue traditionnellement comme une « prérogative royale », les politiques étrangères sont souvent menées au niveau des États centraux. Selon les lois internationales, seuls les États ont la personnalité juridique, contrairement aux entités fédérées. Par conséquent les deux pays sont juridiquement responsables de l'application des traités en droit interne. Mais qu'en est-il pour les entités fédérées ? Ont-elles le droit de signer des traités ou sont-elles consultées quand leurs compétences sont discutées au niveau international ?

Ce chapitre étudie le rôle des entités fédérées dans la politique étrangère des deux États. Cette analyse se base sur deux aspects de la politique étrangère : l'établissement ainsi que la conclusion des traités, et la représentation des entités fédérées à l'étranger ou auprès des organisations internationales. $\mathrm{Ce}$ chapitre est divisé en trois parties. D'abord, la compétence du pouvoir fédéral dans la conclusion des traités est abordée. Ensuite, le deuxième aspect traité est la compétence des entités fédérées dans leurs domaines exclusifs et dans les traités dits « mixtes ». Enfin, la représentation des entités fédérées à l'étranger, au sein des organisations internationales, est étudiée.

\section{a) Les traités dans les compétences exclusives fédérales}

À l'instar du Canada, le Royaume de Belgique est le seul à avoir la personnalité juridique alors que les entités fédérées n'en disposent pas. À ce titre, le siège de l'État fédéral peut librement négocier et signer des traités. Mais avant de signer les traités, l'État fédéral belge doit informer les autres niveaux de gouvernement de ces initiatives. Ce processus d'information passe par l'intermédiaire de la Conférence interministérielle de Politique étrangère (CIPE).

La CIPE constitue un comité de consultation créé en 1993 afin de prévenir ou de résoudre les conflits déjà éclos. Ce comité est composé de délégations des différents pouvoirs, placées sous la présidence du ministre fédéral de politique étrangère. La composition peut varier selon les sujets abordés. Les participants peuvent être les différents ministres aux relations extérieures, des ministres au commerce ou des hauts fonctionnaires. En plus de ces rencontres, différentes réunions consultatives ont lieu au niveau administratif entre fonctionnaires. Parmi les compétences attribuées au CIPE figure la responsabilité de vérifier s'il n'existe pas de conflit de compétences entre les différents niveaux de pouvoir. 
Comme mentionné précédemment, le pouvoir de conclure les traités est la compétence exclusive d'Ottawa. Selon la loi, l'État fédéral n'a pas d'obligation d'informer ou de consulter les provinces avant d'agir sur la scène internationale. En pratique, la situation est différente si le traité touche les compétences déléguées aux provinces. Par le fait d'attribuer la compétence exclusive sans pouvoir aux sous-entités, le système canadien a une approche centralisée. Dans le cas belge, le pouvoir fédéral informe les différentes unités à travers une institution créée pour l'occasion, qui vérifie le respect des compétences attribuées.

\section{b) L'établissement des traités dans les compétences exclusives des sous-entités ou dans les compétences mixtes}

Dans le système politique belge, à l'instar du pouvoir fédéral, les entités fédérées ont le pouvoir de négocier et de signer les traités. Les communautés ont ce pouvoir depuis la révision constitutionnelle de 1970, alors que les régions ont reçu cette compétence plus tard. Les régions et les communautés peuvent négocier et signer des traités dans tous les domaines où ils sont compétents. Ces entités sont également présentes dans les discussions internationales et sur la scène internationale pour négocier directement avec les autres pays.

Afin d'établir une cohérence dans la politique étrangère belge, plusieurs règles ont été fixées en 1993. Avant de commencer une négociation pour un traité, les parties contractantes doivent informer les autres pouvoirs à travers le CIPE qui établit un examen de conformité sur deux critères particuliers. D'abord, il s'agit de s'assurer que la signature du traité n'induise pas un conflit de compétences entre différents niveaux de pouvoir. Ensuite, le CIPE s'engage à vérifier les politiques selon quatre critères ${ }^{8}$ :

- Le parti co-contractant doit être reconnu par le gouvernement belge ;

- La Belgique doit avoir des relations diplomatiques avec la partie adverse ;

- Les relations entre la Belgique et le parti co-contractant ne doivent pas avoir été abolies, suspendues ou compromises ;

- Le traité ne doit pas s'opposer aux obligations internationales ou supranationales de l’État fédéral.

Ces quatre critères sont utilisés pour tous les traités et négociations tenus par toutes les entités au sein de la Belgique : les régions, les communautés et le fédéral. Ces consultations créent une interconnexion entre différents niveaux de pouvoir où une institution centrale veille à la cohésion externe.

Selon la Constitution canadienne confirmée par le Conseil privé en 19379, les affaires étrangères sont de la Couronne, représentée par le gouvernement national. Le

\footnotetext{
${ }^{8}$ Décision du Conseil fédéral des ministres du 18 juin 1993

${ }^{9}$ Arrêt sur le Conventions de travail du Comité judiciaire du Conseil privé de Londres de 1937
} 
pouvoir de négocier et de signer des traités est réservé au fédéral, tandis que les provinces ne peuvent signer que des ententes et des accords. Les provinces peuvent donc conduire certaines négociations, notamment pour des échanges économiques et culturels, et des associations avec des gouvernements de pays ou de régions différentes. Cependant, Ottawa a le pouvoir de répudier ces accords signés par les provinces. Ce droit a été utilisé en 1969 pour annuler l'accord France-Québec sur les satellites de télécommunication. Dans les compétences exclusives des provinces telles que l'éducation, Ottawa n'a d'autre choix que de suivre les lignes directrices données par les provinces ${ }^{10}$ notamment pour des raisons d'application, l’État fédéral n'ayant pas de pouvoir dans ce domaine.

Dans les faits, beaucoup de traités sont dits « mixtes ». Ces traités sont ceux dont les domaines touchent aux compétences de plusieurs niveaux de pouvoir. Au Canada, ce sont les compétences du pouvoir fédéral et des pouvoirs fédérés. Dans le contexte belge, les traités « mixtes » sont ceux qui touchent plus d'un niveau : fédéral-régional, fédéralcommunautaire ou fédéral-communautairerégional.

Tel qu'indiqué précédemment, Ottawa peut être tenu comme responsable pour la non

${ }^{10}$ BROWN, D. M., « Les provinces jouent un rôle dans la politique étrangère canadienne », Fédérations, Numéro spécial 2002, p.11 application des traités. Mais selon la décision rendue par le Conseil privé en 1937, dans le cas des compétences spécifiquement attribuées aux provinces, seuls les acteurs décentralisés sont responsables de l'implémentation en droit interne des traités internationaux conclus par Ottawa. L'État fédéral ne peut pas contraindre juridiquement, ni se substituer aux provinces en cas de non transposition des règles. Il peut arriver que certains traités signés par Ottawa ne soient pas transposés en droit interne. En 1983, le gouvernement fédéral a signé un traité concernant les aspects civils pour les enlèvements d'enfants internationaux. À l'époque de la ratification, seulement quelques provinces ont appliqué cette règle. Certaines provinces ont attendu quelques années avant de l'appliquer ${ }^{11}$.

\section{Pour éviter les conflits liés à la non} application de traités, deux types de stratégie ont été mis en place : la clause fédérale dans les traités internationaux et des mécanismes de consultation. La clause fédérale (aussi appelée « clause canadienne ») affirme que l’État fédéral s'engage à respecter les traités à la limite de ses compétences. Par conséquent, le Canada n'est pas responsable en cas de non application d'un traité par une province. Cette clause a eu deux conséquences directes ${ }^{12}$ : La

\footnotetext{
${ }^{11}$ PAQUIN, S., « Quelle place pour les provinces canadiennes dans les organisations et les négociations internationales du Canada à la lumière des pratiques au sein d'autres fédérations ? ", Canadian Public Administration, Vol. 48, $\mathrm{N}^{\circ} 4$, Winter 2005

${ }^{12}$ Idem
} 
première limite les accords à certaines provinces. Ainsi, lors de la signature de la conférence de La Haye sur le droit international privé, l'État fédéral fit remarquer que la convention ne s'appliquait au début qu'au Manitoba et à Terre-Neuve ${ }^{13}$. La seconde évolution est venue des traités tels l'Accord général sur les tarifs douaniers et le commerce (GATT) et l'Accord de libre-échange nordaméricain (ALENA). Ces accords obligent les signataires à prendre toutes les « mesures raisonnables » pour que ces règles soient appliquées au niveau infra-étatique. Selon le GATT, en cas de réclamation, Ottawa doit montrer qu'il avait fait des efforts sérieux, persistants et convaincants, afin de les transposer en droit interne aux provinces.

La clause fédérale a également poussé l'État fédéral à consulter les provinces afin d'éviter les conflits. Par conséquent, plusieurs processus de consultation ont été établis avec les provinces. En 1967, un organisme de coopération interprovinciale a été constitué pour coordonner la position canadienne dans une rencontre internationale sur l'éducation (l'éducation étant une compétence exclusive des provinces). Depuis 1977, cette organisation établit les délégations canadiennes pour les rencontres internationales portant sur ce domaine ${ }^{14}$.
Dans d'autres domaines, le gouvernement fédéral a créé plusieurs mécanismes de consultation. Ainsi, depuis le « Tokyo Round » de l'Organisation mondiale du commerce (OMC), Ottawa a créé des comités consultatifs pour le commerce international en raison de l'interconnexion complexe des compétences, en ce domaine, entre les provinces et le fédéral. D'autres comités comme le « C-Trade » rassemblent des officiels fédéraux et provinciaux tous les trois mois afin d'échanger des informations et de définir la position canadienne ${ }^{15}$. En pratique, plusieurs comités ont été instaurés pour améliorer la coopération entre les différents niveaux de pouvoir. Mais, ces comités ne sont pas fortement institutionnalisés et Ottawa a parfois pris des décisions sans aucun égard aux avis des provinces ${ }^{16}$.

Dans le cas belge, si une entité désire signer des traités considérés comme « mixtes », elle doit suivre diverses procédures telles que définies par l'accord de coopération du 8 mars 1994. D’abord, elle doit en informer le CIPE qui déterminera si le traité tombe bien dans la catégorie « mixte » et qui doit être impliqué dans la négociation ainsi que la signature. Après avoir informé les différents pouvoirs, les entités qui voient leurs compétences impliquées dans le traité peuvent

\footnotetext{
${ }^{13}$ PAQUIN, S., Loc. Cit.

${ }^{14}$ Idem.
}

\footnotetext{
${ }^{15}$ PAQUIN, S., Loc. Cit.

${ }^{16}$ Idem.
} 
prendre part aux négociations, signer et voter le texte.

Les entités ne sont pas toutes obligées de participer aux négociations et à la signature des traités même si cela tombe sous leurs compétences explicites. Dans ce cas, la partie co-contractante en est informée et se voit indiquée les conséquences légales de cet acte. Ainsi, lors de la convention sur la protection des minorités nationales dans les années 90 , certains désaccords ont surgi entre les différentes entités. Les Communautés française et germanophone étaient d'accord sur le texte alors que la Communauté flamande voyait dans cette convention une menace aux politiques d'homogénéisation linguistique ${ }^{17}$. À la suite de ces désaccords entre les entités ainsi qu'au sein du gouvernement fédéral, cette convention ne fut pas ratifiée par la Belgique.

\section{À l'instar du Canada, l'État fédéral peut} être tenu responsable pour la non-application des traités signés soit par lui-même ou soit par une des entités fédérées. Afin de prévenir les conflits et les condamnations - tel qu'est parfois le cas devant la Cour de J ustice des Communautés européennes - la Belgique a une disposition constitutionnelle depuis 1993. L'article 169 donne à l'État fédéral un pouvoir de substitution qui lui permet d'agir en lieu et

\footnotetext{
${ }^{17}$ LAGASSE, C-E, Le système des relations internationales dans la Belgique fédérale : textes et pratiques, Bruxelles, CRISP, 1997, p. 34
}

place d'une entité fautive. Cette disposition peut être utilisée dans quatre cas précis :

- La Belgique doit avoir été condamnée par une juridiction internationale ou supranationale ;

- L'entité fautive doit avoir été mise en demeure d'agir dans les trois derniers mois ;

- L'entité fautive doit avoir été associée à la procédure de défense ;

- Dans le cas d'un traité « mixte », la totalité de la procédure doit avoir été respectée.

En conclusion, nous constatons qu'en Belgique, toutes les entités ont le pouvoir de signer des traités alors qu'au Canada, seul Ottawa dispose de ce pouvoir et les provinces ont seulement le droit de signer des accords ou des ententes. Une deuxième grande différence est au niveau de l'institutionnalisation. Le Canada a un faible niveau de coordination entre les différentes entités de pouvoir établissant des comités selon les fonctions, alors que la Belgique a institutionnalisé depuis 1993 le comité CIPE afin d'instaurer un dialogue entre les différents niveaux de pouvoir ${ }^{18}$. À ce point de vue, la Belgique a une méthode de fonctionnement plus interconnectée que son homologue canadien qui donne plus de libertés à Ottawa pour décider de la politique étrangère même si les provinces sont consultées. Il est également

\footnotetext{
${ }^{18}$ LAGASSE, C-E, Loc. Cit.
} 
important de noter que la Belgique a gardé un pouvoir central en ayant la possibilité de se substituer à une entité fédérée fautive.

\section{c) Représentation des entités fédérées et fédérales à l'étranger}

Cette partie analyse comment les entités fédérées sont présentes, sur la scène internationale, dans deux domaines : la représentation des intérêts et la conclusion des traités et des accords. Au Canada, six provinces ont une présence à l'étranger : l'Alberta, la Colombie-Britannique, l'Ontario, le Québec, la Nouvelle-écosse et la Saskatchewan. Chaque province s'engage sur la scène internationale pour différentes raisons et de différentes façons. Le Québec accorde beaucoup d'importance aux activités internationales. Pour l'année 2006-2007, le ministère des relations internationales du Québec a eu un budget de 100,3 millions de dollars canadiens, dont 40 millions allant à la représentation de cette province à l'étranger ${ }^{19}$. À titre de comparaison, le budget de l'Ontario, concernant les affaires intergouvernementales (regroupant notamment le Bureau des relations internationales et du protocole), a dépensé l'équivalent de 12,9 millions de dollars canadiens lors de l'année fiscale 2004-200520.

\footnotetext{
${ }^{19}$ Secrétariat du Conseil du trésor du Québec, Budget de dépenses 2006-2007, p. 14-1 Vol. II Crédits des ministères et organismes pris sur

http://www.tresor.gouv.qc.ca/fr/publications/budget/06-07/0607_vol2.pdf

${ }^{20}$ Ministère des finances, Budget des dépenses 2006- 2007, Volume 1, pris sur

http://www.fin.gov.on.ca/french/budget/estimates/200607/volume1/mia.html
}

Les provinces ont différentes façons de conduire les affaires étrangères. Le Québec est très actif pour démontrer ses différences, dont la prééminence du français, à l'étranger ${ }^{21}$ et plus particulièrement depuis la « Révolution tranquille » des années 1960. Cette province s'est souvent démarquée par l'entremise de maintes demandes de siéger à l'UNESCO, la création de la « Maison du Québec » à Paris, l'organisation de plusieurs tournées artistiques, la signature des accords éducatifs et l'organisation des expositions afin de promouvoir ses spécificités22.

Le volontarisme international du Québec s'est maintes fois opposé à Ottawa qui voyait dans cet activisme une menace à l'unité du pays. En 1968, la province du Québec fut invitée à la conférence des ministres francophones de l'éducation au même titre qu'un pays indépendant. Par la suite, Ottawa, craignant que cette province soit reconnue internationalement comme un État souverain ${ }^{23}$, a publié deux documents sur le fédéralisme dans les relations internationales et dans les conférences internationales portant sur l'éducation. Ottawa y a affirmé être le seul à avoir le droit de mener la politique étrangère et de signer les traités.

\footnotetext{
${ }^{21}$ COOPER, A. F., Canadian foreign policy: old habits and new directions, Scarborough, Ont, Prentice-Hall Allyn and BaconCanada, 1997

${ }^{22}$ Idem.

23 DONNEUR, A., Politique étrangère canadienne, Montréal, guérin Universitaire, 1994, p.45.
} 
Les autres provinces agissent dans les affaires internationales avec un autre profil. L'Ontario dispose de plusieurs représentations à l'étranger, principalement aux États-Unis et en Europe. Les initiatives étrangères de cette province se font en informant et en recevant l'accord de la capitale. De plus, l'Ontario essaie d'utiliser les ressources fédérales autant que possible ${ }^{24}$. Dans les faits, mis à part la province du Québec, la plupart des activités internationales des provinces sont complémentaires de celles du gouvernement fédéral ${ }^{25}$.

Pour leur part, les entités belges ont des délégations permanentes à l'étranger. En 1997, la Communauté flamande (incluant la Région flamande avec laquelle elle est fusionnée) avait cinq délégations permanentes : à Washington, La Haye, Vienne, Tokyo et Bruxelles pour la représentation auprès de l’Union Européenne.

La Région wallonne disposait de trois délégations à Washington, Alger et Milan, en plus de quatre autres en coopération avec la Communauté française au Québec, à Kinshasa, à Dakar et à Bruxelles pour la représentation auprès de l’Union Européenne. De plus, la Communauté française disposait de délégations permanentes à Paris, Genève,

\footnotetext{
${ }^{24}$ FELDMAN, E.J., FELDMAN, L.G., "The Impact of Federalism on the Organization of Canadian Foreign Diplomacy”, Publius, vol.14, N4, FALL 1984, p.40.

${ }^{25}$ BROWN, D. M., « Les provinces jouent un rôle dans la politique étrangère canadienne », Fédérations, Numéro spécial 2002, pp. 11-12.
}

Tunis et Prague. La Région Bruxelles-Capitale a seulement une représentation à Bruxelles pour la représentation auprès de l’Union Européenne ${ }^{26}$.

\section{À l'étranger, les diplomates belges} peuvent représenter l'État fédéral ainsi que les entités fédérées. Ils représentent les diverses entités après consultation des attachés communautaires parfois présents dans les représentations du pays. Depuis 1995, les communautés et les régions peuvent nommer un attaché ou un représentant dans une ambassade ou un consulat. Ces attachés sont nommés par leurs entités et sont officiellement diplomates selon le traité de Vienne qui en assure la fonction ${ }^{27}$.

Les entités infra-étatiques peuvent, dans le système belge, représenter le pays dans son ensemble. Il s’agit de cas où les compétences sont exclusivement attribuées aux entités fédérées comme pour la Taalunie (organisation pour la promotion du néerlandais) et pour l'Agence de coopération culturelle et technique, où la Belgique est représentée par la Communauté flamande et la Communauté française ${ }^{28}$. Dans les compétences « mixtes », la participation des entités fédérées dans les enceintes internationales (UN, Benelux,

\footnotetext{
${ }^{26}$ LAGASSE, C-E, Loc. Cit.

${ }^{27}$ Ce système était déjà utilisé par la Communauté française depuis 1986.

${ }^{28}$ La Communauté française est représentée par le commissaire général aux relations internationales de la Communauté française.
} 
Conseil de l'Europe) est permise quand leurs compétences sont à l'agenda de ces réunions. Un ministre régional ou communautaire peut également représenter le pays dans son ensemble au sein de ces institutions et mener la délégation. Le représentant et la position commune sont décidés au sein du CIPE entre les acteurs concernés (ou au P11 pour les affaires européennes). Quand une position commune est défendue par un ministre d'une communauté ou d'une région, il est demandé au ministre conduisant la délégation de défendre la position belge dans son ensemble, plutôt que la position de l'institution de laquelle il émane ${ }^{29}$. Dans le cas où un accord est impossible ou n'a pas été trouvé, le représentant belge doit s'abstenir de voter ${ }^{30}$.

\section{Conclusion}

Le Canada et la Belgique sont souvent cités comme exemples de fédéralisme avec des grandes délégations de pouvoir aux diverses entités. Le premier est une fédération depuis sa création alors que le deuxième est devenu un État fédéral bien plus tard comparativement à son homologue nord-américain. En Belgique, les premières entités ont été créées en 1970 et à travers les différentes réformes entreprises a surgi un État fédéral avec une grande complexité institutionnelle. Ce pays est également parfois utilisé comme exemple pour

\footnotetext{
${ }^{29}$ LAGASSE, N., « Le rôle des régions dans la conduite des relations extérieures belges », Fédérations, vol.2, Nㅜ를 février 2002

${ }^{30}$ Idem.
}

son haut niveau de délégation de pouvoir mais aussi pour son haut niveau de dissociation ${ }^{31}$. En effet, en moins de quarante ans, le pays est passé d'un système unitaire à un système hautement décentralisé, et ce processus continuera probablement dans les prochaines années et notamment après les élections de 2007. Contrairement au cas belge, le Canada semble plus « stable » d'un point de vue institutionnel même si les évolutions institutionnelles sont toujours possibles, notamment en raison de la poussée souverainiste émanant du Québec.

Le but de ce travail était de voir si différentes politiques étrangères sont possibles dans des États fédéraux en observant les cas belge et canadien. Deux approches conviennent à l'analyse : l'approche de la gouvernance à plusieurs niveaux et l'approche centralisée. Ces approches ont permis de voir que la Belgique correspond à une approche à plusieurs niveaux et le Canada correspond davantage à une approche centralisée même si certaines précisions restent à apporter. En Belgique, les compétences en politique étrangère sont partagées. L'État fédéral, les régions et les communautés peuvent conclure des traités

\footnotetext{
${ }^{31}$ KARMIS, D., GAGNON, A-G., « Fédéralisme et identités collectives au Canada et en Belgique : des itinéraires différents, une fragmentation similaire », Canadian Journal of Political Science / Revue canadienne de science politique, Vol.29, N³3, sept. 1996, pp. 435-468 ; LAGASSE, N., « Le fédéralisme Belge : un exemple de fédéralisme de dissociation », presented for the International Forum on Federalism in Mexico Veracruz, 15-17 November 2001. Published on www.forumfed.org
} 
avec relativement peu d'interdits émanant des autres pouvoirs mais avec partage de la prise de décision si le domaine touche les compétences attribuées à d'autres entités. Les provinces canadiennes peuvent signer des accords ou des ententes, mais ceux-ci peuvent être dénoncés par Ottawa. Mais, pour certaines compétences spécifiques comme l'éducation, depuis les années 70, les provinces peuvent envoyer un représentant comme chef de délégation lors de rencontres internationales. Dans les cas expliqués précédemment, le Canada semble correspondre davantage à une vision centralisée de la conduite de la politique étrangère que son homologue outre-Atlantique même si dans certains domaines, à l'instar de l'éducation, la décision semble être plus partagée.

Dans les deux cas étudiés, il y a une grande perte de pouvoir de la part des gouvernements fédéraux. Les deux pays ont des entités fédérées actives sur la scène internationale, mais les envergures de la perte de contrôle du pouvoir central sont différentes. Au Canada, les provinces sont compétentes pour signer des accords et des ententes qui peuvent être annulés par le pouvoir fédéral. De l'autre côté de l'Atlantique, les entités infraétatiques ont la possibilité de signer des traités mais également représenter tout le pays au plan international, empruntant ces compétences du fédéral. Cela se fait avec contrôle ex ante de la part du CIPE qui est également chargé de résoudre d'éventuels conflits ex poste.

En ce qui a trait aux centres de décisions, les entités infra-étatiques sont présentes à l'étranger par différents moyens; elles ont toutes des représentations à l'étranger dans leurs propres délégations ou dans les ambassades et consulats. D'un point de vue de la gestion quotidienne, la Belgique semble offrir une approche plus coopérative et plus institutionnalisée que le Canada. Une explication de cette coopération plus forte pourrait être trouvée dans l'Union Européenne. À ce niveau, très important en terme d'établissement de normes, la Belgique ne peut parler que d'une voix ou ne pas se faire entendre du tout, forçant ainsi les entités à coopérer. 


\section{Bibliographie}

\section{Livres}

COOPER, A. F., Canadian foreign policy: old habits and new directions, Scarborough, Ont, PrenticeHall Allyn and BaconCanada, 1997.

DEHOUSSE, R., Fédéralisme et relations internationales : une réflexion comparative, Bruxelles, Bruylant, 1991.

DONNEUR, A., Politique étrangère canadienne, Montréal, guérin Universitaire, 1994.

HOOGHE, L., MARKS, G., Multi-Level Governance and European Integration, Lanham, Rowman \& Littlefield Publishers, 2001.

LAGASSE, C-E, Le système des relations internationales dans la Belgique fédérale : textes et pratiques, Bruxelles, CRISP, 1997.

VAN DEN BERGH, G., « Les Communautés et Régions belges s'approprient les affaires internationales », Fédérations, vol.3, N¹, février-mars 2003, pp 13-14.

WATTS, R. L., Comparing Federal Systems, Kingston, On, Institute of Intergovernmental Relations, Queen's University, 1999.

\section{$\underline{\text { Articles et conférences }}$}

BROWN, D. M., « Les provinces jouent un rôle dans la politique étrangère canadienne », Fédérations, Numéro spécial 2002, pp. 11-12.

DUCHACEK, I. D., "The International Dimension of Subnational Self-Government”, Publius, vol.14, N², FALL 1984, pp.5-31.

FELDMAN, E.J ., FELDMAN, L.G., "The Impact of Federalism on the Organization of Canadian Foreign Diplomacy", Publius, vol.14, N4, FALL 1984, pp.33-59.

HOOGHE, L., MARKS, G., "Unraveling the Central State, but How? Types of Multi-level Governance", American Political science review, Nº7, 2003, pp.233-243.

KARMIS, D., GAGNON, A-G., « Fédéralisme et identités collectives au Canada et en Belgique : des itinéraires différents, une fragmentation similaire », Canadian J ournal of Political Science / Revue canadienne de science politique, Vol.29, N³, sept. 1996, pp. 435-468.

LAGASSE, N., « Le fédéralisme Belge : un exemple de fédéralisme de dissociation », presented for the International Forum on Federalism in Mexico Veracruz, 15-17 November 2001. Published on www.forumfed.org. 
LAGASSE, N., « Le rôle des Régions dans la conduite des relations extérieures belges », Fédérations, vol.2, $\mathrm{N}^{\circ} 2$, février 2002.

PAQUIN, S., « Quelle place pour les provinces canadiennes dans les organisations et les négociations internationales du Canada à la lumière des pratiques au sein d'autres fédérations ? », Canadian Public Administration, Vol. 48, N4, Winter 2005.

SCHAUS, A., « L'exécution fédérale des obligations internationales : le cas de la Belgique et du Canada », dans GAUDREAULT-DESBIENSJ --F., GÉLINAS, F. (sous la dir. De), Le fédéralisme dans tous ses états : Gouvernance, identité et méthodologie, Cowansville, Canada, 2005, pp.327-348

\section{Documents officiels}

Secrétariat du Conseil du trésor du Québec, Budget de dépenses 2006-2007, p. 14-1Vol. II Crédits des ministères et organismes pris sur http:/ / www.tresor.gouv.qc.ca/ fr/ publications/ budget/ 0607/06-07_vol2.pdf

Secrétariat du Conseil du trésor du Québec, Budget de dépenses 2006-2007, p. 14-1 Vol. II Crédits des ministères et organismes pris sur http:/ / www.tresor.gouv.qc.ca/ fr/ publications/ budget/ 0607/06-07_vol2.pdf 